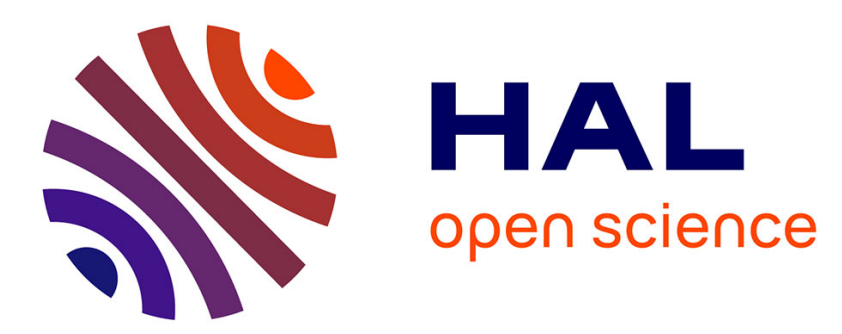

\title{
The effect of planetary rotation on the zigzag instability of co-rotating vortices in a stratified fluid.
}

Pantxika Othéguy, Paul Billant, Jean-Marc Chomaz

\section{To cite this version:}

Pantxika Othéguy, Paul Billant, Jean-Marc Chomaz. The effect of planetary rotation on the zigzag instability of co-rotating vortices in a stratified fluid.. Journal of Fluid Mechanics, 2006, 553 (april), pp.273-281. 10.1017/S0022112005008050 . hal-00021348

\section{HAL Id: hal-00021348 \\ https://hal.science/hal-00021348}

Submitted on 16 Jul 2014

HAL is a multi-disciplinary open access archive for the deposit and dissemination of scientific research documents, whether they are published or not. The documents may come from teaching and research institutions in France or abroad, or from public or private research centers.
L'archive ouverte pluridisciplinaire HAL, est destinée au dépôt et à la diffusion de documents scientifiques de niveau recherche, publiés ou non, émanant des établissements d'enseignement et de recherche français ou étrangers, des laboratoires publics ou privés. 


\title{
The effect of planetary rotation on the zigzag instability of co-rotating vortices in a stratified fluid
}

\author{
By PANTXIKA OTHEGUY†, PAUL BILLANT \\ AND JEAN-MAR C CHOMAZ \\ LadHyX, CNRS, École Polytechnique, F-91128 Palaiseau Cedex, France
}

(Received 4 August 2005 and in revised form 15 November 2005)

This paper investigates the three-dimensional stability of a pair of co-rotating vertical vortices in a rotating strongly stratified fluid. In a companion paper (Otheguy, Chomaz \& Billant 2006), we have shown that such a basic flow in a strongly stratified fluid is affected by a zigzag instability which bends the two vortices symmetrically. In the non-rotating flow, the most unstable wavelength of this instability scales as the buoyancy length and its growth rate scales as the external strain that each vortex induces on the other one. Here, we show that the zigzag instability remains active whatever the magnitude of the planetary rotation and is therefore connected to the tallcolumn instability in quasi-geostrophic fluids. Its growth rate is almost independent of the Rossby number. The most amplified wavelength follows the universal scaling $\lambda=2 \pi F_{h} b \sqrt{\gamma_{1} / R o^{2}+\gamma_{2} / R o+\gamma_{3}}$, where $b$ is the separation distance between the two vortices, $\left(\gamma_{1}, \gamma_{2}, \gamma_{3}\right)$ are constants, $F_{h}$ is the horizontal Froude number and $R o$ the Rossby number $\left(F_{h}=\Gamma / \pi a^{2} N, R o=\Gamma / \pi a^{2} f\right.$, where $\Gamma$ is the circulation of each vortex, $a$ the vortex radius, $N$ the Brunt-Väisälä frequency and $f$ the Coriolis parameter). When $R o=\infty$, the scaling $\lambda \propto F_{h} b$ found in the companion paper Otheguy et al. (2006) is recovered. When $R o \rightarrow 0, \lambda \propto b f / N$ in agreement with the quasi-geostrophic theory. In contrast to previous results, the wavelength is found to depend on the separation distance between the two vortices $b$, and not on the vortex radius $a$.

\section{Introduction}

Oceanic and atmospheric flows are characterized by two important features: a stable density stratification measured by the Brunt-Väisälä frequency $N$, and the planetary rotation measured by the Coriolis parameter $f$. The atmosphere at midlatitudes is typically characterized by a ratio $f / N=0.01$ and the oceans by $f / N=0.1$. A strong stratification reduces the vertical scale to the buoyancy scale $U / N$, where $U$ is the horizontal velocity scale, whereas a rapid rotation tends to increase the vertical scale. Turbulence in stratified fluids is organized into thin horizontal layers (Fincham, Maxworthy \& Spedding 1996; Park, Whitehead \& Gnanadeskian 1994; Godeferd \& Staquet 2003; Waite \& Bartello 2004), while turbulence in rotating fluids is organized into tall eddy columns (Hopfinger, Browand \& Gagne 1982; Baroud et al. 2003). In the joint conditions of rapid rotation and strong stratification (quasi-geostrophic), the vortices have a lens-like shape with a height-to-width aspect ratio $H / L$ proportional to $f / N$ (Charney 1948; Griffiths \& Linden 1981; Dritschel \& de la Torre Juárez 1996). In order to understand the transition between strongly stratified flows and

$\dagger$ Present address: AZTI - Tecnalia, Herrera Kaia Portualdea z/g, 20110 Pasaia, Guipúzcoa, Spain. 
quasi-geostrophic flows as well as the selection of the vertical scale in geophysical flows, we investigate the three-dimensional stability of co-rotating vertical vortices. Such a flow plays a major role in turbulence through the merging process.

We first investigated the three-dimensional stability of a pair of co-rotating vertical vortices in a stratified fluid in the absence of planetary rotation (see the companion paper, Otheguy, Chomaz \& Billant 2006). We observed the elliptic instability when the horizontal Froude number $F_{h}=\Gamma / \pi a^{2} N$ (where $\Gamma$ is the vortex circulation and $a$ the vortex radius) is large: $F_{h}>10$. In contrast, we found that for strong stratification $\left(F_{h}<2.85\right)$ the most unstable three-dimensional instability is a zigzag instability similar to the instability observed for counter-rotating vortices (Billant \& Chomaz 2000). The most unstable wavelength of this instability is proportional to $F_{h} b$, where $b$ is the separation distance between the two vortices. Its growth rate scales like the external strain rate $S=\Gamma / 2 \pi b^{2}$. The instability consists of a symmetric bending of the two vortices which generates horizontal layers of thickness proportional to the buoyancy length. In the successive layers, the vortices are alternately brought closer together or farther from each other every half a wavelength along the vertical direction.

A similar bending instability, named tall-column instability, has been observed by Dritschel \& de la Torre Juárez (1996) and Dritschel (2002) in quasi-geostrophic fluids (strong stratification and rapid rotation) where the most unstable wavelength is proportional to $L_{h} f / N$, where $L_{h}$ is the characteristic horizontal length scale. In the case of two co-rotating vortices, this instability has been shown to enhance or delay (even inhibit) the vortex merging depending on the vertical location.

In order to understand the transition between strongly stratified flows and quasigeostrophic flows and the selection of the vertical length scale for finite Rossby numbers, we investigate herein the effect of planetary rotation on the zigzag instability of a pair of co-rotating vertical Gaussian vortices.

\section{Linear stability analysis}

In Otheguy et al. (2006), a two-dimensional quasi-steady basic state (figure 1) has been obtained numerically using a two-dimensional numerical simulation at a large Reynolds number $(R e=\Gamma / \pi \nu=16000)$ and initialized by two co-rotating axisymmetric vortices with a Gaussian distribution of vorticity. From this initial condition, the two vortices adapt to each other and evolve quickly towards a quasisteady state in the frame of reference rotating at the angular velocity $\Omega_{b}=\Gamma / \pi b^{2}$. The vortex axes are vertical and the ratio of the vortex radius $a$ and the separation distance $b$ between the vortices is $a / b=0.15$. Note that this flow remains a quasisteady basic state in the presence of a Coriolis force (representing the effect of a planetary rotation) since the flow is two-dimensional and incompressible.

The three-dimensional stability analysis of this basic flow is investigated here for a strongly stratified and rotating fluid. The linearized Navier-Stokes equations under the Boussinesq approximation governing the perturbations of velocity $\boldsymbol{u}^{\prime}=\left(u_{x}^{\prime}, u_{y}^{\prime}, u_{z}^{\prime}\right)$, vorticity $\omega^{\prime}$, pressure $p^{\prime}$ and density $\rho^{\prime}$ are

$$
\left.\begin{array}{l}
\frac{\partial \boldsymbol{u}^{\prime}}{\partial t}+\omega_{b} \boldsymbol{e}_{z} \times \boldsymbol{u}^{\prime}+\boldsymbol{\omega}^{\prime} \times \boldsymbol{u}_{b}+\left(2 \Omega_{b}+f\right) \boldsymbol{e}_{z} \times \boldsymbol{u}^{\prime}=-\nabla\left(\frac{p^{\prime}}{\rho_{0}}+\boldsymbol{u}_{b} \cdot \boldsymbol{u}^{\prime}\right)-g \frac{\rho^{\prime}}{\rho_{0}} \boldsymbol{e}_{z}+v \Delta \boldsymbol{u}^{\prime}, \\
\nabla \cdot \boldsymbol{u}^{\prime}=0, \\
\frac{\partial \rho^{\prime}}{\partial t}+\boldsymbol{u}_{b} \cdot \nabla \rho^{\prime}-\frac{\rho_{0}}{g} N^{2} u_{z}^{\prime}=D \Delta \rho^{\prime},
\end{array}\right\}
$$




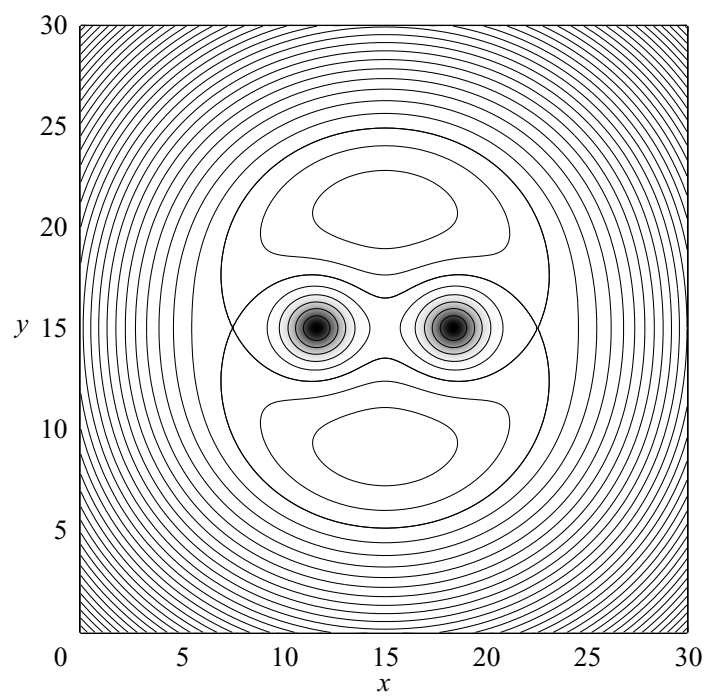

FIGURE 1. Streamlines (contour lines) and vorticity field (background grey levels) of the basic state. The vorticity contours are superimposed on the streamlines showing that this basic state is quasi-steady.

where $\boldsymbol{u}_{b}$ is the velocity of the basic state, $\omega_{b}$ its vorticity, $\boldsymbol{e}_{z}$ the unit vector in the upward vertical direction, $f$ the Coriolis parameter equal to twice the local planetary rotation rate in the $f$-plane approximation, $\rho_{0}$ a constant reference density, $g$ the acceleration due to gravity, $v$ the kinematic viscosity, $N$ the Brunt-Väisälä frequency and $D$ the molecular diffusivity of the stratifying agent. As the basic state is uniform along the $z$-axis, the perturbations can be written as follows:

$$
\left[\boldsymbol{u}^{\prime} ; \boldsymbol{\omega}^{\prime} ; p^{\prime} ; \rho^{\prime}\right](x, y, z, t)=[\boldsymbol{u} ; \boldsymbol{\omega} ; p ; \rho]\left(x, y, k_{z}, t\right) \mathrm{e}^{\mathrm{i} k_{z} z}+\text { c.c. },
$$

where $k_{z}$ is the vertical wavenumber and c.c. denotes the complex conjugate. A pseudo-spectral code has been used to integrate equation (2.1) for each value of $k_{z}$. The size of the computational domain is large in order to minimize the effect of the periodic boundary conditions: $L_{x}=L_{y}=30 a$. The number of collocation points is $M_{x}=M_{y}=256$ and the time step is $\delta t=0.01 \pi a^{2} / \Gamma$. The perturbation of velocity $\boldsymbol{u}$ is initialized by a divergence-free white noise and after integrating equation (2.1) for a sufficiently long time, the most unstable eigenmode dominates the perturbation (see Otheguy et al. 2006 for further details).

In the present paper, this stability analysis is performed mainly for a single Froude number $F_{h}=\Gamma / \pi a^{2} N=1$ as a function of the Rossby number $R o$ defined as

$$
R o=\frac{\Gamma}{\pi a^{2} f} .
$$

The Reynolds number is $R e=16000$ and the Schmidt number is $S c=v / D=1$.

Figure 2 represents the evolution of the growth rate of the zigzag instability as a function of the vertical wavenumber $k_{z}$ for various cyclonic rotations for which the Rossby number is positive, and anticyclonic rotations for which the Rossby number is negative. When $R o=\infty$, the zigzag instability destabilizes a large band of vertical wavenumbers $0 \lesssim k_{z} b \lesssim 18$. The maximum growth rate has been shown in Otheguy et al. (2006) to be approximately equal to twice the external strain $S=\Gamma / 2 \pi b^{2}$ and the most amplified wavenumber is around $k_{z} b=10$ for $F_{h}=1$. When planetary rotation 


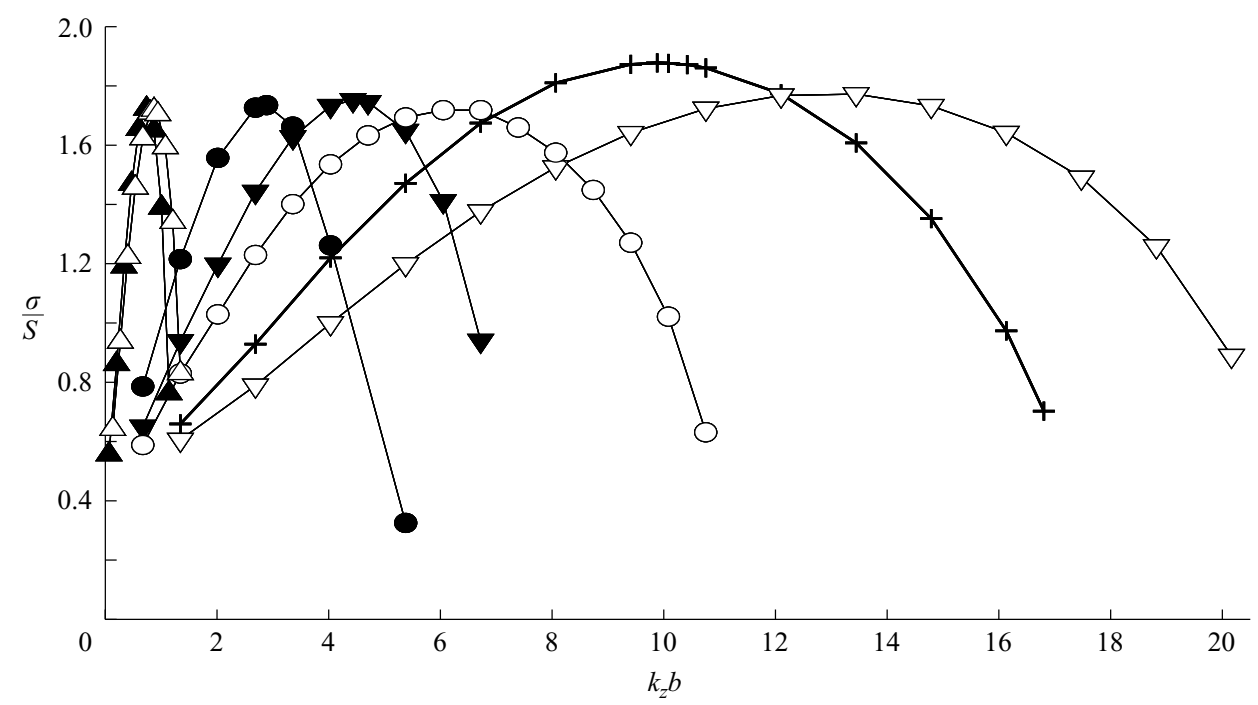

FIGURE 2. Growth rate of the zigzag instability $\sigma$ normalized by the strain rate $S=\Gamma / 2 \pi b^{2}$ plotted against the vertical wavenumber $k_{z}$ scaled by the separation distance $b$ for $F_{h}=1$, $R e=16000, a / b=0.15$ and $R o=\infty(+), R o= \pm 5(\mathbf{\nabla}, \nabla), R o= \pm 2.5(\bullet, \bigcirc)$ and $R o= \pm 0.5$ $(\Lambda, \triangle)$. Cyclonic rotations are represented by filled symbols whereas anticyclonic rotations are represented by open symbols.

is present, all the curves keep the same shape with a maximum growth rate varying very little, but the most amplified wavenumber varies considerably depending on the Rossby number $R o$. For large $R o(R o= \pm 5)$, the effects of cyclonic and anticyclonic rotations are different. The cyclonic rotation tends to shift the curve relative to the $R o=\infty$ curve towards smaller wavenumbers while anticyclonic rotation displaces the curves towards higher wavenumbers. For smaller $R o(R o= \pm 2.5)$, both cyclonic and anticyclonic rotations shift the curves towards smaller wavenumbers but not by the same amount. For $R o= \pm 0.5$, the effect of anticyclonic rotation is the same as for cyclonic rotation: the two growth rate curves are almost superimposed.

Figure 3 shows the maximum growth rate and the corresponding most amplified vertical wavenumber as a function of the Rossby number. As already seen in figure 2, the maximum growth rate is almost independent of the planetary rotation (figure $3 a$ ) although it is slightly smaller for positive Rossby numbers and small negative Rossby numbers than for $R o=\infty$. For anticyclonic rotations $R o \lesssim-1$, the maximum growth rate is slightly enhanced. In contrast, the most unstable wavenumber varies strongly with Ro (figure $3 b$ ). For small Rossby numbers, the most unstable wavenumber increases linearly with $R o$ with the same slope for cyclonic and anticyclonic rotations. For positive Rossby numbers, the wavenumber continues to increase monotonically and tends to the non-rotating value $k_{z m}(R o=\infty)$ as the Rossby number increases. In contrast, the most unstable wavenumber reaches a maximum for negative Rossby numbers and then decreases asymptotically towards $k_{z m}(R o=\infty)$.

Strikingly, the entire dependence of the most unstable wavenumber $k_{z m}$ on the Rossby number $R o$ for a constant horizontal Froude number $\left(F_{h}=1\right)$ follows the law

$$
k_{z m} b=f(R o)=\left(\frac{\gamma_{1}}{R o^{2}}+\frac{\gamma_{2}}{R o}+\gamma_{3}\right)^{-1 / 2}
$$



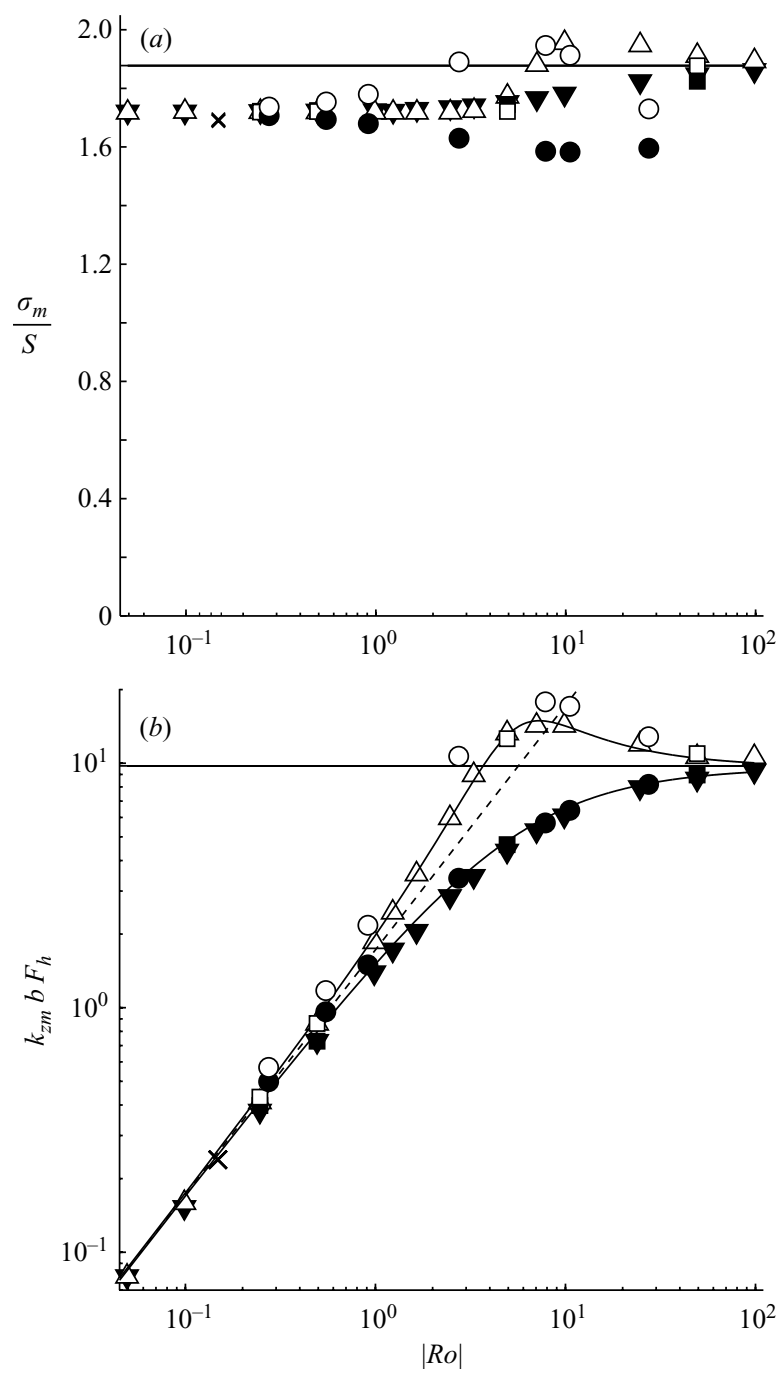

Figure 3. (a) Maximum growth rate normalized by the external strain rate $\sigma_{m} / S$ and (b) most amplified wavenumber $F_{h} k_{z m} b$ plotted against the Rossby number $R o$ for $R e=16000$. The asymptotic values $\sigma_{m}(R o=\infty)$ and $k_{z m}(R o=\infty)$ are represented by a horizontal line. The dashed line in $(b)$ represents the high-rotation-rate approximation: $k_{z m} b F_{h}=1.74 R o$. The solid lines display the fit $k_{z m}=f(R o) / F_{h} b$. The symbols $\boldsymbol{\Delta}, \nabla$ represent $F_{h}=1$ and $a / b=0.15 ; \mathbf{\square}, \square$ represent $F_{h}=0.5$ and $a / b=0.15$ and $\bullet, \bigcirc F_{h}=1$ and $a / b=0.21$ for cyclonic rotation (filled symbols) and anticyclonic rotation (open symbols). The symbols $\times$ correspond to the point $R o=0.15$ and $F_{h}=0.015$, which are typical parameter values characterizing most mesoscale eddies in the ocean.

where the constants $\gamma_{1}=0.33, \gamma_{2}=0.09, \gamma_{3}=0.01$ are the same both for cyclonic $(R o>0)$ and anticyclonic $(R o<0)$ rotations. The function $f(R o)$ is always real even for negative $R o$.

Alternatively, when the horizontal Froude number $F_{h}$ is varied, it has been shown in Otheguy et al. (2006) that $k_{z m} \propto 1 / F_{h} b$ when there is no rotation $(R o=\infty)$. Without further argument, these two scaling laws can be combined in the general form $k_{z m}=F\left(R o, F_{h}\right) / F_{h} b$ where $F$ is a priori a function of $F_{h}$ and $R o$. However, the 
scaling analysis of Billant \& Chomaz (2001) for strongly stratified rotating flows has proved that the function $F\left(R o, F_{h}\right)$ does not depend on $F_{h}$ when $F_{h} \ll 1$ whatever the Rossby number $R o$ is. This implies

$$
k_{z m}=\frac{f(R o)}{F_{h} b}
$$

for any Rossby number and any small Froude number. This scaling law has been checked by considering the case $F_{h}=0.5$ for finite Rossby numbers. Figure $3(b)$ shows that the scaled wavenumber $k_{z m} F_{h} b$ does indeed follow the curve $f(R o)$ strikingly. Another value of the ratio $a / b$ has also been considered, $a / b=0.21$, for $F_{h}=1$. Varying $a / b$ corresponds to varying the strain $S$. Again, the wavenumber follows the same universal scaling law. The growth rate normalized by the strain also remains approximately the same although it is slightly reduced, in particular for large Rossby numbers, as already noted in Otheguy et al. (2006) for $R o=\infty$.

In the limit of small Rossby numbers, the most unstable wavenumber becomes proportional to the ratio of the Rossby number to the Froude number:

$$
k_{z m}=\frac{1}{\sqrt{\gamma_{1}}} \frac{R o}{F_{h} b}=\frac{1}{\sqrt{\gamma_{1}}} \frac{N}{f b},
$$

i.e. to the ratio $N / f$ in agreement with the quasi-geostrophic theory (Charney 1948). However, a crucial difference with the scaling laws reported previously (Dritschel \& de la Torre Juárez 1996) in quasi-geostrophic fluid is that the most unstable wavelength $\lambda_{m}=2 \pi / k_{z m} \approx 3.6 b f / N$ is proportional to the separation distance $b$ and not to the vortex radius $a$. This dependence on $b$ has also been noted by Dritschel (2002) and might explain why different constants of proportionality have been reported in quasigeostrophic turbulence since the wavelength was scaled by $\mathrm{fa} / N$ (McWilliams 1990; Reinaud, Dritschel \& Koudella 2003; McWilliams, Weiss \& Yavneh 1999).

The distribution of vorticity inside each vortex is also expected to affect the constant of proportionality (here equal to 3.6) but not the scaling of the wavelength on $b$. Here, a Gaussian vorticity profile has been chosen while in Dritschel \& de la Torre Juárez (1996), the vorticity is taken constant inside the vortex core. A further possible reason for these differences in the literature is that the height of the computational domain is often smaller than the most amplified wavelength so that the top and bottom boundary conditions might influence the wavelength selection.

Figure 4 displays the growth rate curves of figure 2 plotted as a function of the rescaled wavenumber $k_{z} b / f(R o)$. The collapse is very good for all the wavenumbers but better for small wavenumbers. This means that the scaling is not only valid for the most unstable wavenumber but also for all the wavenumbers, i.e. $\sigma\left(k_{z}, b, R o, F_{h}\right) \approx$ $S \tilde{\sigma}\left(k_{z} b F_{h} / f(R o)\right)$, where $\tilde{\sigma}$ is a single function of the rescaled wavenumber.

Figure 5 shows the vertical vorticity of the eigenmode for different Rossby numbers and for the most unstable wavenumber. The same dipolar shape in each vortex core with the same orientation (angle between the dipole axis and the line joining the vortices) is observed for all the Rossby numbers. As explained in Otheguy et al. (2006) this shape corresponds to a bending of each vortex obliquely in opposite directions, with no deformation of their core. Together with the results found in Otheguy et al. (2006) where the Rossby number was infinite and the Froude number was varied, from this we conclude that the self-similarity property of the zigzag instability on the single variable $k_{z} F_{h} b / f(R o)$ is therefore a fundamental property of the growth rate as well as the shape of the eigenmode of the instability. 


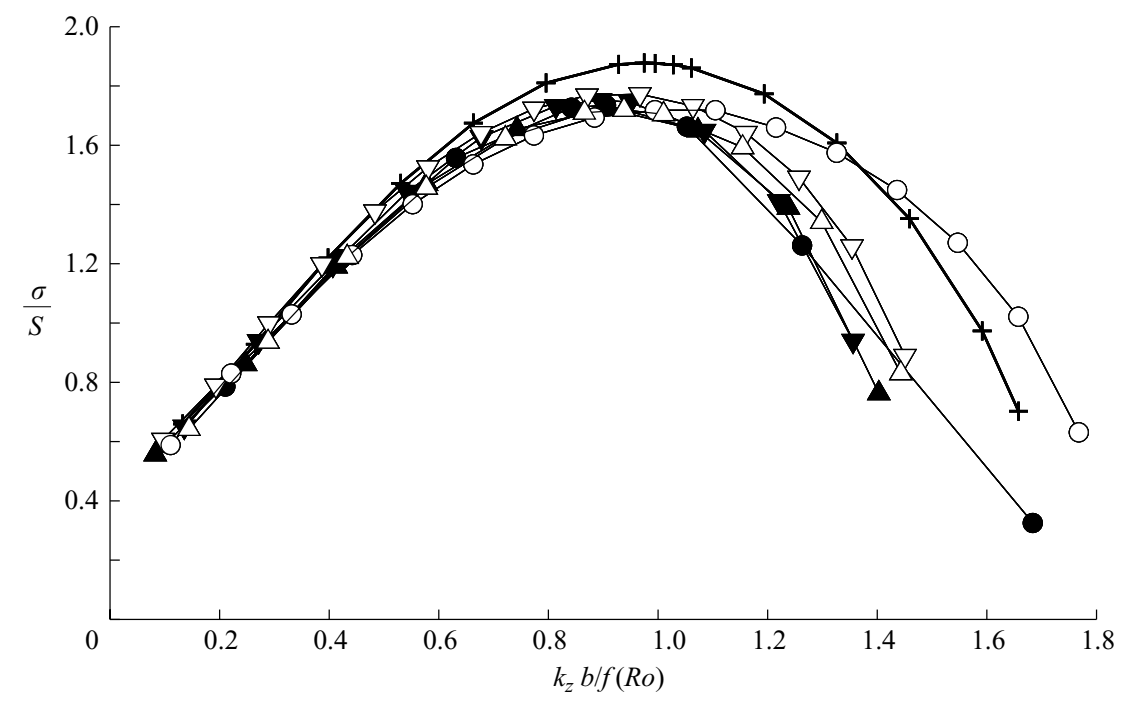

FIGURE 4. Growth rate of the zigzag instability normalized by the strain rate $\sigma / S$ plotted against the vertical wavenumber $k_{z}$ rescaled by $b / f(R o)$ for $F_{h}=1, R e=16000, a / b=0.15$ and $R o=\infty(+), R o= \pm 5(\nabla, \nabla), R o= \pm 2.5(\bullet, O)$ and $R o= \pm 0.5(\mathbf{\Lambda}, \triangle)$. Cyclonic rotations are represented by filled symbols whereas anticyclonic rotations are represented by open symbols.

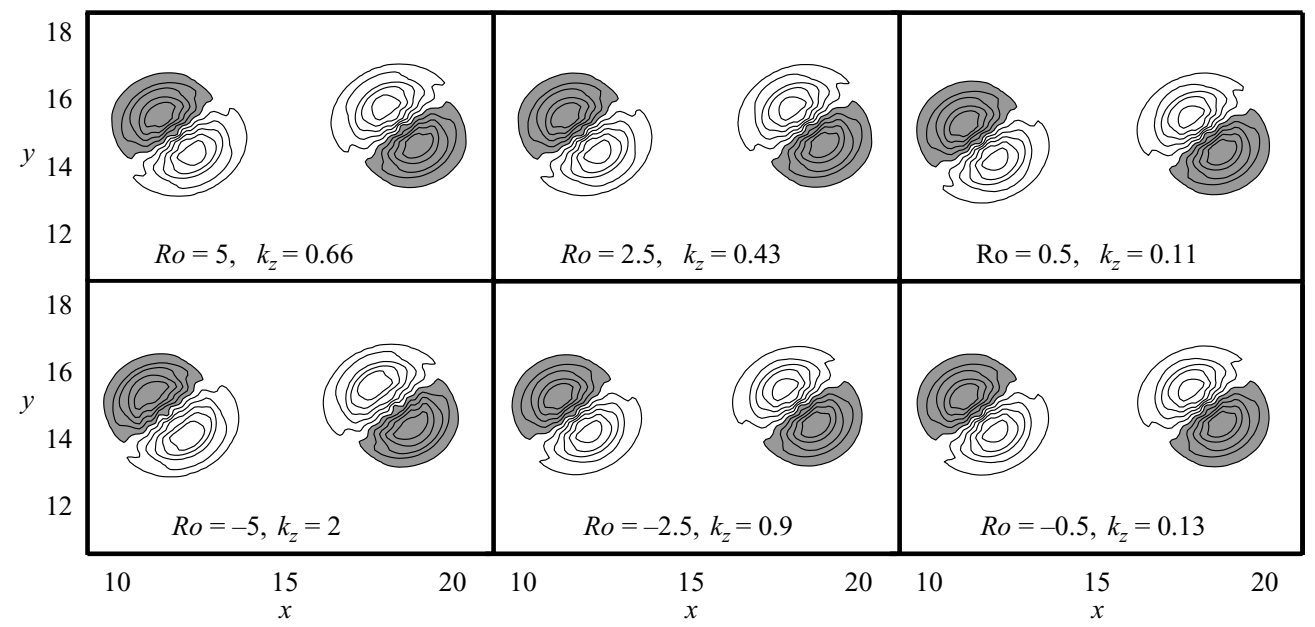

FiguRE 5. Vertical vorticity of the eigenmode for different Rossby numbers for the most unstable wavenumber. The Froude number is $F_{h}=1$ and the Reynolds number $R e=16000$. Shaded regions indicate positive vorticity.

\section{Discussion}

Oceanic and atmospheric flows at large scales $(>500 \mathrm{~km})$ have both a small Froude number and a small Rossby number so that the quasi-geostrophic approximation is valid. The typical value $f / N=0.1$ for the oceans at mid-latitudes corresponds to a wavelength $\lambda=0.36 b$ for the zigzag or tall-column instability while in the atmosphere, the typical value is $f / N=0.01$ leading to $\lambda=0.036 b$. With solid boundary conditions, the zigzag or tall-column instability would be observed on co-rotating Gaussian columnar vortices of equal intensity in the atmosphere or oceans only if the instability 
wavelength were smaller or at least of the same order as the fluid height $H: \lambda \lesssim H$. This puts a strong constraint on the size of the vortices on which the zigzag or tall-column instability could be observed. However, it should be noted that the zigzag or tall-column instability is a long-wavelength instability which destabilizes all the wavelengths in the band $\left[\lambda_{\text {cutoff }}, \infty\right]$ so that large vortices can still be affected if $H \gtrsim \lambda_{\text {cutoff. }}$. Furthermore, in the case of free boundary conditions, the vortices can be bent even if a wavelength does not fit within the fluid height.

For an ocean $5 \mathrm{~km}$ deep, the maximum value of the separation distance $b$ allowing one entire wavelength to be observed is $b=14 \mathrm{~km}$ meaning that the zigzag instability will display one entire wavelength only for vortices of scale about $10 \mathrm{~km}$ and smaller. For example, mesoscale eddies observed in the Gulf Stream, Mediterranean sea (Richardson 1993), Hawaiian sea (Chavanne et al. 2002) or in the Bay of Biscay (Pingree \& Le Cann 1992) have a typical radius around $150 \mathrm{~km}$, and are characterized by typical $R o=0.15$ and $F h=0.015$. The zigzag or tall-column instability scaling then predicts (figure $3 b) \lambda \approx 0.36 b>O(100) \mathrm{km}$, a far too large value to fit a wavelength in the ocean depth since $b$ should be larger than $2 a$. It should be mentioned however that this estimate has been obtained by assuming the constant ratio $f / N=0.1$ while in practice this ratio can be smaller and varies spatially.

Smaller vortices observed in the Ligurian Sea (Marullo, Salusti \& Viola 1985) or in the Southern California Bight (DiGiacomo \& Holt 2001) have a radius of about $5 \mathrm{~km}$, a typical velocity of $0.5 \mathrm{~m} \mathrm{~s}^{-1}$ and are approximately $150 \mathrm{~m}$ deep. They are localized mainly in the thermocline where the stratification is approximately $N \approx 10^{-2} \mathrm{~s}^{-1}$ so that $f / N \approx 0.01$. The Rossby and Froude numbers corresponding to these eddies are about $R o \approx 2$ and $F_{h} \approx 0.02$. The scaling $(2.5)$ can be applied and yield $\lambda \approx 0.04 b$. Most eddies are typically separated by a distance $O(10 \mathrm{~km})$ which gives half a wavelength of order $\lambda / 2 \sim O(200 \mathrm{~m})$, in agreement with their typical depth. The zigzag or tall-column instability is probably very active since cyclonic vortices are found to be numerous in small areas (DiGiacomo \& Holt 2001), promoting vortex interactions and thereby the instability.

\section{Conclusion}

We have shown that the zigzag instability remains active on a co-rotating pair of vortices in the presence of planetary rotation. It is therefore of the same physical nature as the tall-column instability shown by Dritschel \& de la Torre Juárez (1996) (see also Dritschel, de la Torre Juárez \& Ambaum 1999; Dritschel 2002; Reinaud \& Dritschel 2005 ) to cause the breakdown of columnar vortices in the presence of strain in a quasi-geostrophic fluid. Its growth rate is proportional to the strain rate $S=\Gamma / 2 \pi b^{2}$ and almost independent of the Rossby number but the vertical wavelength varies according to the universal scaling $\lambda=2 \pi F_{h} b \sqrt{\gamma_{1} / R o^{2}+\gamma_{2} / R o+\gamma_{3}}$, i.e. from $\lambda / b \propto F_{h}$ for $R o=\infty$ to $\lambda / b \propto F_{h} / R o \propto f / N$ for $R o \ll 1$. This instability therefore should play a major role for a wide range of geophysical scales since its existence requires only that the Froude number is low. A striking result is that the wavelength is proportional to the distance between the vortices and independent of the vortex radius. This might explain why different scaling laws in term of the vortex radius have been reported for quasi-geostrophic fluids (Dritschel \& de la Torre Juárez 1996; McWilliams 1990; Reinaud et al. 2003; McWilliams et al. 1999). Because of this dependence on the separation distance $b$, the wavelength of the zigzag or tall-column instability in the quasi-geostrophic regime is relatively large compared to the height of the atmosphere or the oceans except when the vortices are close to one another. 
We wish to thank gratefully David Dritschel, Colm-cille Caulfield and Anne-Virginie Salsac for fruitful discussions and Daniel Guy for technical assistance. This work is supported by IDRIS (CNRS) for computational facilities under project No.41722.

\section{REFERENCES}

Baroud, C. N., Plapp, B. B., She, Z.-S. \& Swinney, H. L. 2003 Scaling in three-dimensional and quasi-two-dimensional rotating turbulent flows. Phys. Fluids 15, 2091-2104.

Billant, P. \& Chomaz, J.-M. 2000 Experimental evidence for a new instability of a vertical columnar vortex pair in a strongly stratified fluid. J. Fluid Mech. 418, 167-188.

Billant, P. \& Chomaz, J.-M. 2001 Self-similarity of strongly stratified inviscid flows. Phys. Fluids 13, $1645-1651$.

Charney 1948 On the scale of atmospheric motion. Geofys. Publ. 17, 2,1-17.

Chavanne, C., Flament, P., Lumpkin, R., Dousset, B. \& Bentamy, A. P. 2002 Scatterometer observations of wind variations induced by oceanic islands: Implications for wind-driven ocean circulation. Can. J. Remote Sensing 28, 466-474.

DiGiacomo, P. M. \& Holt, B. 2001 Satellite observations of small coastal ocean eddies in the southern california bight. J. Geophys. Res. 106, 22521-22543.

Dritschel, D. 2002 Vortex merger in rotating stratified flows. J. Fluid Mech. 455, 83-101.

Dritschel, D. \& DE LA TORRe JuÁrez, M. 1996 The instability and breakdown of tall columnar vortices in a quasi-geostrophic fluid. J. Fluid Mech. 328, 129-160.

Dritschel, D., De la Torre Jú́rez, M. \& Ambaum, M. H. P. 1999 The three-dimensional vortical nature of atmospheric and oceanic turbulent flows. Phys. Fluids 11, 1512-1520.

Fincham, A. M., Maxworthy, T. \& Spedding, G. R. 1996 Energy dissipation and vortex structure in freely decaying, stratified grid turbulence. Dyn. Atmos. Oceans 23, 155-169.

Godeferd, F. S. \& Staquet, C. 2003 Statistical modelling and direct numerical simulations of decaying stably stratified turbulence. Part 2. Large-scale and small-scale anisotropy. J. Fluid Mech. 486, 115-159.

Griffiths, R. W. \& Linden, P. F. 1981 The stability of vortices in a rotating stratified fluid. J. Fluid Mech. 105, 283-316.

Hopfinger, E. J., Browand, F. K. \& GaGne, Y. 1982 Turbulence and torsional waves in a rotating tank. J. Fluid Mech. 125, 505-534.

Marullo, S., Salusti, E. \& Viola, A. 1985 Observations of a small-scale baroclinic eddy in the Ligurian sea. Deep-Sea Res. A 32, 215-222.

McWilliams, J. C. 1990 The vortices of geostrophic turbulence. J. Fluid Mech. 219, 387-404.

McWilliams, J. C., Weiss, J. \& Yavneh, I. 1999 The vortices of homogeneous geostrophic turbulence. J. Fluid Mech. 401, 1-26.

Otheguy, P., Сhomaz, J.-M. \& Billant, P. 2006 Elliptic and Zigzag instabilities on co-rotating vortices in a stratified fluid. J. Fluid Mech. 553, 253-272.

Park, Y.-G., Whitehead, J. \& Gnanadeskian, A. 1994 Turbulent mixing in stratified fluids: layer formation and energetics. J. Fluid Mech. 279, 279-311.

Pingree, R. D. \& Le Cann, B. 1992 Three anticylonic slope water oceanic eddies (swoddies) in the southern bay of biscay. Deep-Sea Res. 39, 1147-1175.

ReINAUd, J. \& Dritschel, D. 2005 The critical merger distance between two co-rotating quasigeostrophic vortices. J. Fluid Mech. 522, 357-381.

Reinaud, J., Dritschel, D. \& Koudella, C. R. 2003 The shape of vortices in quasi-geostrophic turbulence. J. Fluid Mech. 474, 175-192.

Richardson, P. L. 1993 Tracking ocean eddies. Am. Sci. 81, 261-271.

Waite, M. \& Bartello, P. 2004 Stratified turbulence dominated by vortical motion. J. Fluid Mech. 517, 281-308. 